\title{
Chest physical therapy for children hospitalised with acute pneumonia: a randomised controlled trial
}

\author{
C Paludo, L Zhang, C S Lincho, D V Lemos, G G Real, J A Bergamin
}

Department of Maternal and Child Health, Federal University of Rio Grande, Rio Grande, Brazil

Correspondence to: Dr L Zhang, Department of Maternal and child Health, Federal University of Rio Grande, Rio Grande, Brazil 96010-140; zhanglinjie63@yahoo.com.br

Received 31 July 2007 Accepted 31 January 2008 Published Online First 14 February 2008

\section{ABSTRACT}

Background: The indication for chest physical therapy as an adjunct to the treatment of children hospitalised with acute pneumonia remains controversial and there is a lack of robust scientific evidence for the effectiveness of this modality in these patients.

Methods: A randomised controlled trial was conducted in two tertiary hospitals in southern Brazil. Children aged 29 days to 12 years hospitalised with pneumonia between February and October 2006 were recruited; 51 were randomly allocated to the intervention group (chest physical therapy plus standard treatment for pneumonia) and 47 to the control group (standard treatment for pneumonia alone). The primary outcome was time to clinical resolution. The secondary outcomes were length of stay in hospital and duration of respiratory symptoms and signs.

Results: There were no significant differences in terms of median time to clinical resolution (4.0 vs 4.0 days, $p=0.84$ ) and median length of hospital stay (6.0 vs 6.0 days, $p=0.76$ ) between the intervention and control groups. The intervention group had a longer median duration of coughing (5.0 vs 4.0 days, $p=0.04$ ) and of rhonchi on lung auscultation (2.0 vs 0.5 days, $p=0.03$ ) than the control group.

Conclusions: Chest physical therapy as an adjunct to standard treatment does not hasten clinical resolution of children hospitalised with acute pneumonia and may prolong duration of coughing and rhonchi.

The indication for chest physical therapy as an adjunct to the treatment of children hospitalised with acute pneumonia remains controversial. On the one hand, chest physical therapy has been, and continues to be, widely applied to these patients in paediatric practice based on beliefs of the potential benefits of this modality in evacuating inflammatory exudates and tracheobronchial secretions, removing airway obstruction, reducing airway resistance, enhancing gas exchange and reducing the work of breathing..$^{1-3}$ On the other hand, there is a lack of robust scientific evidence for the effectiveness of chest physical therapy in children hospitalised with pneumonia. The British Thoracic Society guidelines for managing pneumonia in childhood recommend that chest physical therapy is not beneficial and should not be performed in children with pneumonia; ${ }^{4}$ however, these recommendations are based mainly on the results of two randomised controlled trials, one in adults and one in children. ${ }^{56}$ Moreover, the validity of this old clinical trial in children is questionable due to the small sample size, exclusion of patients with pneumonia of presumed bacterial origin and inadequate randomisation and blinding. ${ }^{6}$ More data are therefore needed from high quality randomised trials to make a more precise decision on the use of chest physical therapy for acute pneumonia in childhood.

This randomised controlled trial was conducted to assess the effectiveness of chest physical therapy in children hospitalised with acute pneumonia. We hypothesised that chest physical therapy as an adjunct to standard treatment could hasten the clinical resolution of acute pneumonia in paediatric inpatients.

\section{METHODS}

\section{Study setting}

The study was conducted in the paediatric wards of two tertiary hospitals: the 30-bed teaching hospital of the Federal University of Rio Grande and the 30-bed Hospital da Santa Casa de Rio Grande. These are two unique hospitals in the city of Rio Grande in southern Brazil, covering a population of approximately 200000 . The ethics committee of the Federal University of Rio Grande (Rio Grande, RS, Brazil) and the ethics committee of the Hospital da Santa Casa de Rio Grande (Rio Grande, RS, Brazil) approved the study.

\section{Participants}

Children aged 29 days to 12 years hospitalised with a diagnosis of acute pneumonia were assessed for eligibility for the study. Patients were included in the study if they met all of the following clinical and radiological diagnosis criteria for pneumonia: ${ }^{4}$ (1) presence of coughing and/or fever; (2) tachypnoea, defined as respiratory rate above agedependent upper limits: $<2$ months: 60 breaths/ min (bpm); 2-12 months: 50 bpm; $>1-5$ years: 40 bpm; $>5$ years: $30 \mathrm{bpm}$; (3) consolidations and/or infiltrates associated or not with other findings compatible with pneumonia on the chest radiograph. All chest radiographs were interpreted routinely in the Department of Radiology and reviewed by a senior paediatric pulmologist (ZL). Any disagreement was resolved by discussion with a reference radiologist. Patients who needed a chest drain, had haemodynamic instability, bone fragility or rib fractures and any other contraindication to chest physical therapy were excluded. ${ }^{1}$ The recruitment of participants occurred between February and October 2006. The parents or legal guardians of each child gave written informed consent before enrolment in the study.

\section{Study design and protocol}

This study was a randomised controlled clinical trial. Simple randomisation was performed from a table of random numbers. The recruited 
participants were randomly allocated to the intervention group or the control group. The intervention group received chest physical therapy twice daily plus standard treatment for pneumonia until discharge and the control group received standard treatment for pneumonia alone. Each session of chest physical therapy took about $30 \mathrm{~min}$ and consisted of postural drainage, thoracic squeezing, chest percussion, vibration, cough stimulation and aspiration of secretions (if necessary). ${ }^{156}$ The positions for postural drainage were guided by the chest radiograph to allow more effective drainage of tracheobronchial secretions and inflammatory exudates in the most affected areas. ${ }^{16}$ The same certified physiotherapist (CP) administered chest physical therapy to all patients.

The standard treatment for pneumonia in each patient was administered by the attending paediatrician based on the recommendations of the Brazilian guidelines for the diagnosis and treatment of pneumonia. ${ }^{7}$ It included antibiotic therapy, fluid therapy and oxygen therapy if needed. The decision to discharge from hospital was also made by the attending pediatrician. All attending pediatricians were blinded to group assignment and study protocol.

The patients were assessed at enrolment and every day between 17.00 and $18.00 \mathrm{~h}$ by one of four investigators using a pre-codified questionnaire. The same investigator was responsible for assessment of each patient from enrolment to discharge. Patient assessment included respiratory symptoms, respiratory signs (respiratory rate, chest indrawing, nasal flaring, cyanosis and lung auscultation) and arterial oxygen saturation measured by pulse oximetry. All four investigators received adequate training in assessment of the patient, especially in measuring respiratory rate and arterial oxygen saturation, in observing chest indrawing and in lung auscultation. The standard technique for measurement of respiratory rate and arterial oxygen saturation was the same as previously reported. ${ }^{8}$ The definition of adventitious sounds on lung auscultation was based on the recommendations of the 1985 International Symposium on Lung Sounds. ${ }^{9}$ The interobserver agreement between the four investigators and a senior paediatric pulmologist $(\mathrm{ZL})$ regarding respiratory signs (tachy-pnoea, chest indrawing, rhonchus, wheezes, fine crackles and coarse crackles) was evaluated and kappa indexes of 0.53 , $0.63,0.48,0.70,0.60$ and 0.47 , respectively, were achieved. The daily maximum body temperature was noted by the investigators based on the patient's nursing record. The patient's axillary temperature was measured by the attending nurses every $3 \mathrm{~h}$ throughout the hospital stay. All investigators and nurses were blinded to group assignment and study protocol. Different schedules were arranged for the investigators and physiotherapist to avoid their chance encounter at a patient's bedside.

\section{Outcome measures}

The primary outcome was time to clinical resolution which was defined as the number of days needed for a patient to achieve the following clinical parameters: afebrile (daily maximum body temperature $<37.5^{\circ} \mathrm{C}$ ), absence of severe signs (chest indrawing, nasal flaring, cyanosis), normal respiratory rate and arterial oxygen saturation $\geqslant 95 \%$. Secondary outcomes were length of hospital stay (in days) and persistence of respiratory symptoms and signs (fever, cough, wheezing, tachypnoea, chest indrawing, adventitious sounds on lung auscultation and arterial oxygen saturation $<95 \%$ ) (in days).

\section{Sample size}

The sample size was determined on the assumption that the mean (SD) time to clinical resolution in the control group was 6 (1.5) days. ${ }^{6}$ To detect a clinically relevant reduction of 1 day in the time to clinical resolution, 47 participants were needed in each group (two-sided $\alpha$ of $5 \%$ and power of $90 \%$ ).

\section{Statistical analysis}

Statistical analysis was performed using the Stata Version 8.0 program (Stata Corporation, Texas, USA). The $\chi^{2}$ test was used for analysis of dichotomous data and the unpaired Student $t$ test or Mann-Whitney rank sum test were used for analysis of continuous data as appropriate. Analyses were based on the intention-to-treat principle. In the additional analysis we excluded the possible cases of acute bronchiolitis (ie, patients aged $<2$ years who presented with wheeze on lung auscultation

Figure 1 Flow diagram of the trial.

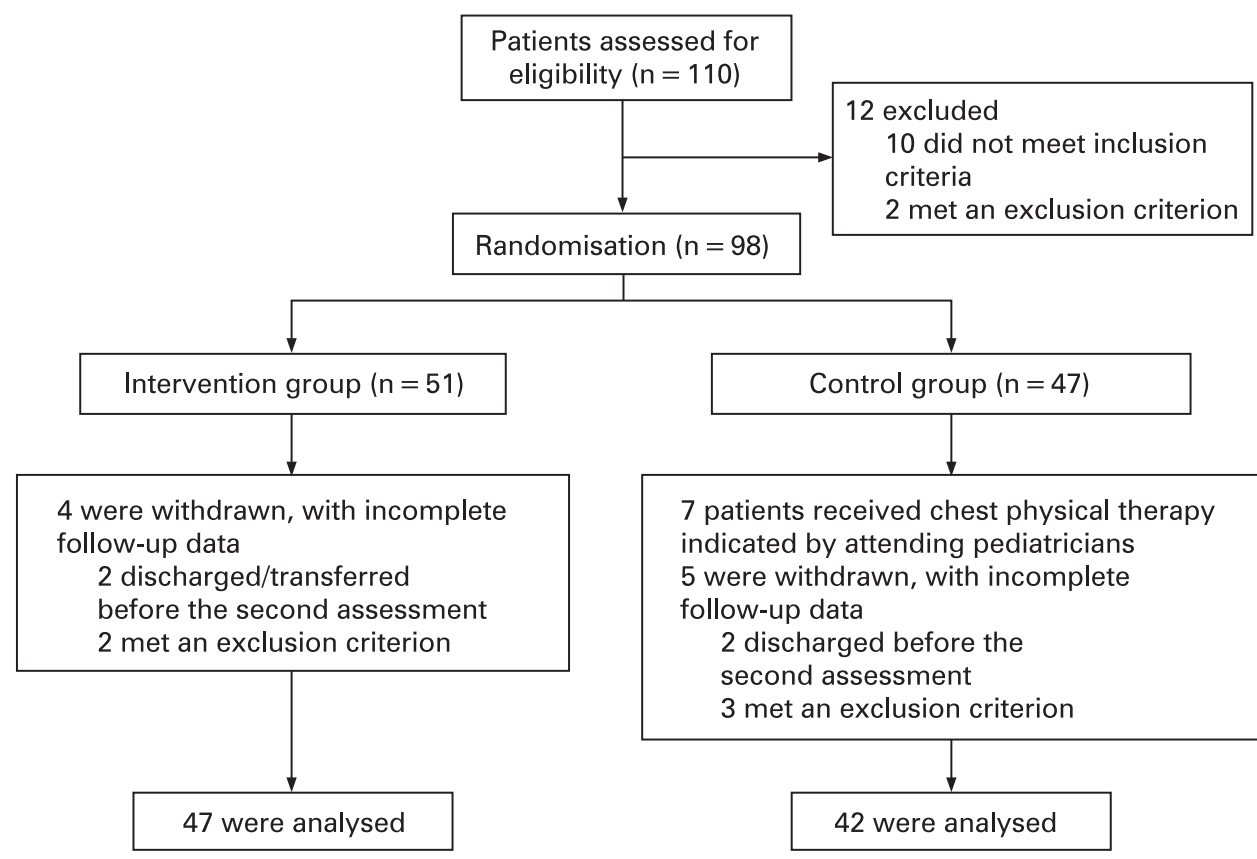


Table 1 Baseline characteristics of the 89 patients

\begin{tabular}{|c|c|c|c|}
\hline Characteristic & $\begin{array}{l}\text { Intervention group } \\
(\mathrm{n}=47)\end{array}$ & $\begin{array}{l}\text { Control group } \\
(\mathrm{n}=42)\end{array}$ & p Value \\
\hline Age (months) & 44.0 (31.6 to 56.4$)$ & $32.2(22.5$ to 41.9$)$ & 0.14 \\
\hline Male sex & $29(61.7 \%)$ & $24(57.1 \%)$ & 0.66 \\
\hline Low birth weight $(<2500 \mathrm{~g})$ & $5(10.6 \%)$ & $4(9.5 \%)$ & 0.86 \\
\hline Prematurity ( $<37$ weeks) & $8(17.0 \%)$ & $6(14.2 \%)$ & 0.72 \\
\hline Maternal smoking & $17(36.1 \%)$ & $17(40.4 \%)$ & 0.67 \\
\hline \multicolumn{4}{|l|}{$\begin{array}{l}\text { Respiratory symptoms and } \\
\text { signs }\end{array}$} \\
\hline Coughing & $46(97.8 \%)$ & $38(92.6 \%)$ & 0.24 \\
\hline Fever & $45(95.7 \%)$ & $37(90.2 \%)$ & 0.30 \\
\hline Parent's reported wheezing & $31(65.9 \%)$ & $24(58.5 \%)$ & 0.47 \\
\hline Respiratory rate (bpm) & $45.0(40.9$ to 49.1$)$ & $45.8(41.6$ to 50.1$)$ & 0.78 \\
\hline Chest indrawing & $24(51.0 \%)$ & $22(53.6 \%)$ & 0.80 \\
\hline Fine crackles & $16(34.0 \%)$ & $11(26.8 \%)$ & 0.46 \\
\hline Coarse crackles & $17(36.1 \%)$ & $19(46.3 \%)$ & 0.33 \\
\hline Rhonchi & $9(19.2 \%)$ & $6(14.6 \%)$ & 0.57 \\
\hline Wheezes & $13(27.6 \%)$ & $5(12.2 \%)$ & 0.07 \\
\hline \multicolumn{4}{|l|}{ Chest radiographic findings } \\
\hline Consolidation & $39 / 45(86.7 \%)$ & $35 / 39(89.7 \%)$ & 0.66 \\
\hline Hyperinflation & $6 / 45(13.3 \%)$ & $5 / 39(12.8 \%)$ & 0.95 \\
\hline Pleural effusion & $5 / 45(11.1 \%)$ & $6 / 39(15.4 \%)$ & 0.56 \\
\hline Arterial oxygen saturation & 95.0 (94.3 to 95.7$)$ & 95.7 (95.0 to 96.4 ) & 0.15 \\
\hline Antibiotic therapy & $47(100 \%)$ & $42(100 \%)$ & 1.00 \\
\hline
\end{tabular}

Values expressed as mean $(95 \% \mathrm{Cl})$ or $\mathrm{n}(\%)$.

at enrolment. An explanatory analysis was also performed to verify the potential changes in the results. A two-tailed $p$ value of $<0.05$ was considered statistically significant.

\section{RESULTS}

One hundred and ten children were assessed for eligibility during the study period and 98 were included in the trial (fig 1). Of the 98 patients recruited, 51 were randomly allocated to the intervention group and 47 to the control group. Table 1 summarises the baseline characteristics of the 89 patients. There were no significant differences between the intervention and control groups in terms of the baseline characteristics.

Table 2 compares the clinical evolution in the intervention and the control groups. The intervention group had a longer median duration of coughing (5.0 vs 4.0 days, $p=0.04$ ) and of rhonchi on lung auscultation ( 2.0 vs 0.5 days, $p=0.03$ ) than the control group. There were no significant differences between the two groups in the other parameters of clinical evolution.

No significant changes were observed in the results when an additional analysis (excluding 12 patients who were aged $<2$ years who presented with wheeze at enrolment) and an explanatory analysis (excluding 7 patients in the control group who received chest physical therapy indicated by attending pediatricians) were performed.

\section{DISCUSSION}

This randomised trial failed to confirm that chest physical therapy as an adjunct to standard treatment hastens the clinical resolution of children hospitalised with acute pneumonia. The time to clinical resolution and the length of hospital stay were similar in the intervention and control groups. Moreover, this study showed that patients who received chest physical therapy had had prolonged duration of coughing and rhonchi on lung auscultation. However, the persistence of these respiratory symptoms and signs may not necessarily represent an unfavourable clinical evolution. Coughing might be induced by a physiotherapist since it is an essential component of chest
Table 2 Effects of chest physical therapy on clinical evolution of children hospitalised with acute pneumonia

\begin{tabular}{llll}
\hline Outcomes & $\begin{array}{l}\text { Intervention group } \\
(\mathbf{n}=\mathbf{4 7})\end{array}$ & $\begin{array}{l}\text { Control group } \\
(\mathbf{n}=42)\end{array}$ & p Value \\
\hline Time to clinical resolution & $4.0(2.0-7.0)$ & $4.0(3.0-6.0)$ & 0.84 \\
Length of hospital stay & $6.0(4.0-9.0)$ & $6.0(4.0-8.0)$ & 0.76 \\
Time to normal respiratory rate & $3.0(0-7.0)$ & $3.0(1.0-6.0)$ & 0.75 \\
Time to normal arterial oxygen & $1.0(0-2.0)$ & $0.5(0-2.0)$ & 0.98 \\
saturation ( $\geqslant 95 \%)$ & & $4.0(2.0-6.0)$ & 0.28 \\
Time to normal lung auscultation & $4.0(3.0-6.0)$ & $1.0(0-3.0)$ & 0.78 \\
Duration of fever & $2.0(0-2.0)$ & $4.0(3.0-6.0)$ & 0.04 \\
Duration of coughing & $5.0(4.0-8.0)$ & $1.0(0-3.5)$ & 0.29 \\
Duration of parent's reported & $1.5(0-5.0)$ & & \\
wheezing & & $0(0-2.0)$ & 0.72 \\
Duration of fine crackles & $0(0-2.0)$ & $1.0(0-3.0)$ & 0.83 \\
Duration of coarse crackles & $2.0(0-4.0)$ & $0(0-4.0)$ & 0.62 \\
Duration of wheezes & $0(0-5.0)$ & $0.5(0-2.0)$ & 0.03 \\
Duration of rhonchi & $2.0(0-4.0)$ & $2.0(0-3.0)$ & 0.75 \\
Duration of chest indrawing & $2.0(0-3.0)$ &
\end{tabular}

Number of days expressed as median (interquartile range).

physical therapy. Rhonchus, a typical "secretion sound" on lung auscultation, might be prolonged by chest physical therapy as it could dislodge tracheobronchial secretions and produce this adventitious sound. In this sense, chest physical therapy might effectively mobilise tracheobronchial secretions in this group of children but failed to modify the clinical evolution of acute pneumonia assessed by time to clinical resolution, length of hospital stay and persistence of other individual clinical parameters such as fever, respiratory rate, chest indrawing and arterial oxygen saturation.

One clinical trial reported no beneficial effect of chest physical therapy in children hospitalised with viral pneumonia in spite of serious methodological limitations mentioned previously. ${ }^{6}$ Moreover, prolonged fever was observed in the intervention group. A similar finding was reported by another randomised trial in adults with primary pneumonia. ${ }^{5}$ It was speculated that the spread of infiltrates and physical activity associated with chest physical therapy might explain the prolonged fever in patients receiving this treatment. In the present trial the intervention group had a longer median duration of fever than the control group (2.0 vs 1.0 days) but this difference was not statistically significant $(p=0.78)$.

The data derived from this randomised trial support the recommendations of the British Thoracic Society guidelines ${ }^{4}$ that chest physical therapy is not beneficial in children with acute pneumonia and should not be performed in these patients. However, these recommendations may not be suitable for special groups of children with acute pneumonia, such as those with impaired mucociliary clearance or those with complications such as atelectasis and pleural effusion needing a chest drain. Future randomised trials should focus on these special groups of patients.

Some methodological aspects of this trial deserve special comment. First, no placebo is available to blind those involved in a trial of physical therapy and it may be the source of performance and observation bias. ${ }^{10}$ To minimise these potential biases, all investigators who assessed the patients and all attending paediatricians and nurses were blinded to the group assignment and study protocol. Second, no standard protocol of physical therapy is well defined for special respiratory disease and the "art" of this modality may introduce a number of personal and uncontrollable factors. In this trial the protocol of physical therapy was defined a priori based on the experiences 
of the previous studies and on the potential benefits of each component on acute pneumonia. ${ }^{156}{ }^{11}$ To avoid inter-individual variation caused by the "art" of physical therapy, the same physiotherapist administered the treatment to all patients. Third, the outcomes currently used to assess the effectiveness of chest physical therapy-such as radiolabelled aerosols, chest radiographs, sputum volume, oxygen saturation and pulmonary function testing-are considered unreliable or impractical, especially in a paediatric population. ${ }^{2}$ We used the time to clinical resolution as the primary outcome since we expected that chest physical therapy could hasten the clinical resolution of children hospitalised with acute pneumonia. This variable consists of various relevant and relatively accurate signs which reflect the severity and evolution of pneumonia. ${ }^{4712}$ This outcome may therefore be more reliable and objective than the length of hospital stay or duration of any individual symptom or sign to represent clinical evolution of acute pneumonia in childhood.

Acknowledgements: The authors thank Raúl Mendoza-Sassi for advice on statistical analysis.

Funding: CP, CSL and JAB received grants from the following Brazilian government research support agencies: the Coordination for the Improvement of Higher Education Personnel (CAPES), the National Council for the Scientific and Technologic Development (CNPq) and the Research Assistance Fund of Rio Grande do Sul (FAPERGS).

Competing interests: None.
Ethics approval: The ethics committee of the Federal University of Rio Grande (Rio Grande, RS, Brazil) and the ethics committee of the Hospital da Santa Casa de Rio Grande (Rio Grande, RS, Brazil) approved the study. The parents or legal guardians of each child gave written informed consent before enrolment.

\section{REFERENCES}

1. Balachandran A, Shivbalan S, Thangavelu S. Chest physiotherapy in pediatric practice. Indian Pediatr 2005;42:559-68.

2. Wallis C, Prasad A. Who needs chest physiotherapy? Moving from anecdote to evidence. Arch Dis Child 1999:80:393-7.

3. Chalumeau M, Foix-L'Helias L, Schinmann P, et al. Rib fractures after chest physiotherapy for bronchiolitis or pneumonia in infants. Pediatr Radiol 2002;32:644-7.

4. British Thoracic Society Standards of Care Committee. BTS guidelines for the management of community acquired pneumonia in childhood. Thorax 2002;57/Suppl 1):i1-24.

5. Britton S, Bejstedt M, Vedin L. Chest physiotherapy in primary pneumonia. BMJ 1985;290:1703-4.

6. Levine A. Chest physical therapy for children with pneumonia. J Am Osteopath Assoc 1978;78:122-5.

7. Sociedade Brasileira de Pneumologia e Tisologia. I Consenso brasileiro de pneumonias. J Pneumol 1998;24:101-8.

8. Zhang L, Ferruzzi E, Bonfanti T, et al. Long and short-term effect of prednisolone in hospitalized infants with acute bronchiolitis. J Paediatr Child Health 2003:39:548-51.

9. Mikami R, Murao M, Cugell DW, et al. International symposium on lung sounds. Synopsis of proceedings. Chest 1987;92:342-5.

10. Greenhalgh T. Assessing the methodological quality of published papers. BMJ 1997:315:305-8.

11. Oberwaldner B. Physiotherapy for airway clearance in paediatrics. Eur Respir $J$ 2000; 15:196-204.

12. Palafox M, Guiscafré $\mathrm{H}$, Reyes $\mathrm{H}$, et al. Diagnostic value of tachypnoea in pneumonia defined radiologically. Arch Dis Child 2000;82:41-5. 